\title{
Miranda mediates asymmetric protein and RNA localization in the developing nervous system
}

\author{
Alison J. Schuldt, ${ }^{1}$ Jan H.J. Adams, ${ }^{1}$ Catherine M. Davidson, ${ }^{1}$ David R. Micklem, ${ }^{1}$ Jim Hasel off, ${ }^{2}$ \\ Daniel St Johnston, ${ }^{1}$ and Andrea H. Brand ${ }^{1,3}$ \\ ${ }^{1}$ Wellcome/CRC Institute and Department of Genetics, Cambridge CB2 1QR, UK; ${ }^{2}$ Medical Research Council (MRC) \\ Laboratory of Molecular Biology, Cambridge CB2 2QH, UK
}

\begin{abstract}
Neuroblasts undergo asymmetric stem cell divisions to generate a series of ganglion mother cells (GMCs). During these divisions, the cell fate determinant Prospero is asymmetrically partitioned to the GMC by Miranda protein, which tethers it to the basal cortex of the dividing neuroblast. Interestingly, prospero mRNA is similarly segregated by the dsRNA binding protein, Staufen. Here we show that Staufen interacts in vivo with a segment of the prospero $3^{\prime}$ UTR. Staufen protein and prospero RNA colocalize to the apical side of the neuroblast at interphase, but move to the basal side during prophase. Both the apical and basal localization of Staufen are abolished by the removal of a conserved domain from the carboxyl terminus of the protein, which interacts in a yeast two-hybrid screen with Miranda protein. Furthermore, Miranda colocalizes with Staufen protein and prospero mRNA during neuroblast divisions, and neither Staufen nor prospero RNA are localized in miranda mutants. Thus Miranda, which localizes Prospero protein, also localizes prospero RNA through its interaction with Staufen protein.
\end{abstract}

[Key Words: RN A localization; asymmetric segregation; Miranda; Staufen; Prospero; nervous system]

Received April 3, 1998; revised version accepted April 27, 1998.

As the nervous system develops, cellular diversity increases dramatically as thousands of neurons are born, and each takes on its own identity. An efficient method for generating diversity is to ensure that when a cell divides, each of its daughters assumes a distinct identity. This is most simply achieved by the unequal partitioning of cell fate determinants at each cell division. Such a mechanism is used in yeast to differentiate mother cells from their daughters (Bobola et al. 1996; Sil and Herskowitz 1996; Long et al. 1997; Takizawa et al. 1997), in early Caenorhabditis elegans development to distinguish the sister cells arising from the first embryonic divisions (for review, see N elson and Grindstaff 1997), and in the CN S of both vertebrates (Chenn and M cConnell 1995; Zhong et al. 1996) and Drosophila (Rhyu et al. 1994; Hirata et al. 1995; Knoblich et al. 1995; Spana and Doe 1995; Spana et al. 1995).

In the Drosophila embryonic CNS, neural precursors (or neuroblasts) divide in a stem cell lineage, gi ving rise to a series of smaller daughter cells called ganglion mother cells (GMCs). At least two cell fate determinants, the homeodomain protein Prospero (Doe et al. 1991; Vaessin et al. 1991) and the membrane-associated protein N umb (U emura et al. 1989; Rhyu et al. 1994), are preferentially segregated to the GMC at cell division. To achieve this, the subcellular distribution of both proteins is regulated during the cell cycle (Rhyu et al. 1994; Hirata et al. 1995; Knoblich et al. 1995; Spana and Doe 1995; Spana et al. 1995). At prophase, N umb and Prospero form a tight crescent on the basal side of the neuroblast such that, as the GMC buds off, the two proteins are asymmetrically segregated to the daughter cell. $\mathrm{N}$ umb remains at the cortex in the GMC, whereas Prospero is released and enters the nucleus. Prospero specifies the GMC fate by repressing neuroblast-specific genes and activating GMC-specific genes (Doe et al. 1991; Vaessin et al. 1991; Matsuzaki et al. 1992). Although the role of Numb in directing GMC fates is unclear, it has been shown to specify the fate of one of the two daughters of the MP2 precursor (Spana et al. 1995). $\mathrm{N}$ umb segregates to the dMP2 daughter in which it inhibits the $\mathrm{N}$ otch signal transduction pathway (Spana and Doe 1996).

Prospero interacts with a protein called Miranda, which anchors it to the cell membrane (IkeshimaKataoka et al. 1997; Shen et al. 1997). In the absence of Miranda, Prospero is never localized at the cortex of the neuroblast and, as a result, enters the nucleus in both neuroblasts and GMCs. Miranda al so forms a basal crescent in neuroblasts at prophase and is segregated with Prospero and N umb into the GMC at cell division. Once 
in the GMC, Miranda is rapidly degraded (IkeshimaKataoka et al. 1997; Shen et al. 1997) and Prospero is released.

The prospero mRNA is also asymmetrically localized in mitotic neuroblasts, and segregates into the GMC at cell division ( $\mathrm{Li}$ et al. 1997; Broadus et al. 1998). This process has been shown to require a protein, Staufen, which contains five repeats of a putative dsRN A binding domain (dRBD; St Johnston et al. 1992). Staufen was first identified through its role in establishing the anteriorposterior asymmetry of the Drosophila oocyte (St Johnston 1995). Staufen associates with oskar mRN A to mediate its transport to the posterior of the oocyte, where the mRNA is anchored and translated (Ephrussi et al. 1991; Kim-Ha et al. 1991; St Johnston et al. 1991). Staufen also anchors bicoid mRN A at the anterior of the embryo (Ferrandon et al. 1994). In staufen mutants, the loss of asymmetry causes head defects and elimination of the abdomen (Schüpbach and Wieschaus 1989).

Of the five potential dsRNA binding domains in Staufen, dRBD 2 and dRBD 5 do not bind dsRN A in vitro and dRBD3 binds, but without sequence specificity (Bycroft et al. 1995; S. Grunert and D. St Johnston, unpubl.). As specific RNA binding has not been demonstrated in vitro by use of the full-length protein, an in vivo RNA injection assay was developed to demonstrate that Staufen mediates the localization of bcd through interaction with its $3^{\prime}$ UTR (Ferrandon et al. 1994). When the bcd 3' UTR is injected into early embryos, it recruits the endogenous Staufen into ribonucleoprotein particles that localize to the astral microtubules, supporting the observation that RN A transport in the oocyte depends on microtubules (Pokrywka and Stephenson 1991; Clark et al. 1994; Pokrywka and Stephenson 1995).

Although Staufen mediates RN A localization both in the oocyte and in the nervous system, there are several obvious differences between the two processes. RN A Iocalization in neuroblasts appears to depend on actin microfilaments rather than on microtubules (Broadus et al. 1998). Whereas RNA localization is essential in establishing polarity in the oocyte, it appears to be expendable in the nervous system and no obvious phenotype is observed in a staufen mutant (Li et al. 1997; Broadus et al. 1998; A.J. Schuldt, C.M. Davidson, and A.H. Brand, unpubl.). In the oocyte, mRNA translation relies on localization (Kim-Ha et al. 1995; Webster et al. 1997), whereas the translation and distribution of Prospero and Numb proteins is unaffected in staufen mutant embryos ( $\mathrm{Li}$ et al. 1997; Broadus et al. 1998; A.J. Schuldt, C.M. Davidson, and A.H. Brand, unpubl.). Rather than acting as a primary mechanism for differentiating neuroblast and GMC cell fates, asymmetric RN A local ization appears to support protein localization, ensuring that sufficient Prospero is either transported to, or can be translated by, the GMC (Broadus et al. 1998). This may be particularly important as GMCs do not transcribe prospero themselves. In support of this, the alteration in GMC cell fates that occurs when Prospero protein, or its acti vity, is reduced, is exacerbated by the loss of Staufen (Broadus et al. 1998). The combination of protein and RN A localization ensures the rapid and efficient segregation of Prospero to the GMC.

Staufen is not the only protein involved in RN A localization in the nervous system. Inscuteable, a novel membrane-associated protein with a putative $\mathrm{SH} 3$ binding site (Kraut and Campos-Ortega 1996), is required for prospero mRN A to move from the apical side of the neuroblast to the basal side at mitosis ( $\mathrm{Li}$ et al. 1997). Inscuteable orients the mitotic spindle in neuroblasts and ensures that Numb and Prospero protein crescents localize on the basal side of the cell, rather than randomly (Kraut et al. 1996).

Inscuteable is able to bind to Staufen in a yeast twohybrid screen, and may anchor Staufen at the apical cortex (Li et al. 1997). However, there is only a partial loss of Staufen Iocalization in the absence of Inscuteable. Furthermore, al though both Inscuteable and Staufen are required for the basal localization of prospero mRN A, both proteins were found only on the apical side of the cell ( $\mathrm{Li}$ et al. 1997). Although there are now conflicting accounts of the subcellular distribution of Staufen (Broadus and Doe 1997; Li et al. 1997; Broadus et al. 1998), it is still unclear what mediates the transition of the prospero mRN A from the apical side of the neuroblast to the basal cortex.

We have investigated the factors requi red for the subcellular distribution of Staufen and prospero mRN A. We find that Staufen can interact, in vivo, with the prospero 3' UTR. We have identified the region of Staufen re qui red for its subcellular distribution, and show that this region interacts di rectly with M iranda. M iranda col ocalizes with Staufen protein and prospero mRN A throughout the cell cycle, concentrating first on the apical side of the cell at interphase and then forming a basal crescent at mitosis. Furthermore, Miranda is required in vivo for the correct localization of Staufen protein and prospero mRN A. Thus, Miranda coordinates the subcellular distribution of both the Prospero protein and its mRNA, through a direct interaction with the prospero mRNAbinding protein, Staufen.

\section{Results}

Staufen binds to the 3' UTR of the prospero mRNA in vivo

Previous reports suggested that Staufen can interact directly with the 3' UTR of prospero mRNA in vitro ( $\mathrm{Li}$ et al. 1997). However, the fragment of Staufen used in these experiments, dRBD3, can bind in vitro to any doublestranded RNA longer than $11 \mathrm{bp}$, including adenovirus VA 1 RN A and the U 1 and $U 2$ snRN As (St Johnston et al. 1992; S. Grunert and D. St Johnston, unpubl.). Furthermore, as full-length Staufen protein is extremely insoluble, specific RNA binding cannot be assayed in vitro. For these reasons, Ferrandon et al . (1994) devel oped an in vivo assay for RNA binding, which is based on the observation that Staufen forms ribonucleoprotein particles (RN Ps) with RNAs with which it interacts. When bcd 
mRNA, but not a control dsRNA, is injected into the early embryo, Staufen is specifically recruited into particles at the site of injection.

To assay RNA binding in vivo, we injected the prospero 3' UTR into embryos expressing a green fluorescent protein (GFP)-Staufen protein fusion and monitored the formation of Staufen RN P particles. The full-length prospero 3' UTR forms particles (Fig. 1a), as does the bicoid 3' UTR, but not the coding region of the prospero mRN A, nor VA1 RN A, even though it is able to form an extended secondary structure (data not shown). These RNPs are associated with the nuclei of the precellular embryo, and move with them to the cortex at stage 4 (Fig. 1d). However, unlike the RN P particles formed between Staufen and the bcd 3' UTR (Ferrandon et al. 1994), the Staufen/prospero 3' UTR particles do not associate with the astral microtubules (Fig. 1e). Similar results are observed when the prospero $3^{\prime}$ UTR is injected into embryos expressing wild-type Staufen (detected with anti-Staufen antibodies), rather than a GFP fusion, whereas VA 1 RN A does not recruit Staufen (A.J. Schuldt and A.H. Brand, unpubl.).

To further map the region of the prospero 3' UTR with which Staufen interacts, we injected either the $3^{\prime}$ half of the UTR (Fig. 1b), or the 5' half into embryos (Fig. 1c). Whereas the 3' segment recruits Staufen into RNPs within 5-10 min of injection, the $5^{\prime}$ segment does so only slightly, if at all, after 20-30 min. Therefore, the region of the prospero $m R N A$ recognized by Staufen lies in the terminal 650 bases of the mRNA.

\section{Staufen colocalizes with Prospero}

throughout the cell cycle

Previous reports describing the subcellular distribution of Staufen have been conflicting. Li et al. (1997) reported that Staufen remains on the apical side of the neuroblast throughout the cell cycle, suggesting that it cannot directly mediate formation of the basal crescent of prospero mRN A. Conversely, Staufen is only observed on the basal side of neuroblasts cultured in vitro (Broadus and Doe 1997). Our results concur with those recently reported by Broadus et al. (1998) and demonstrate that Staufen colocalizes with Prospero protein at all stages of the cell cycle. In embryos, we observe that Staufen is concentrated on the apical side of the neuroblast at interphase, then forms a crescent on the basal side of the cell in prophase, where it remains through mitosis before partitioning to the GMC at division (Fig. $2 a, c, e, g)$. A similar subcellular distribution is seen in living embryos (A.H. Brand, unpubl.). This dynamic pattern of localization shows Staufen to be correctly placed to bind the prospero mRNA throughout the cell cycle, and to mediate its segregation into the GMC (see Fig. 6a,b, below).

\section{Staufen localization requires dRBD5}

Experiments in the oocyte have identified two regions of Staufen that are required for its function during oogenesis: a 99-amino-acid region in the middle of dRBD2, and the carboxy-terminal 157 amino acids that include dRBD5 (Micklem 1998). As neither the dRBD2 insert nor the carboxy-terminal domain binds dsRNA in vitro (S. Grunert and D. St Johnston, unpubl.), these two regions may be involved in some other aspect of Staufen function. When Staufen protein that lacks either the dRBD2 insert or the carboxy-terminal domain is expressed maternally, it can only partially rescue the abdominal defects caused by Staufen null mutations
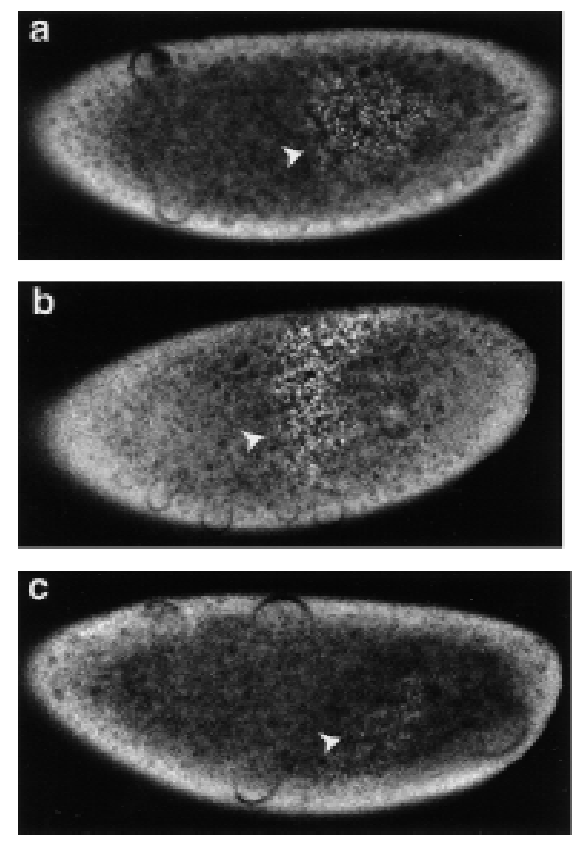
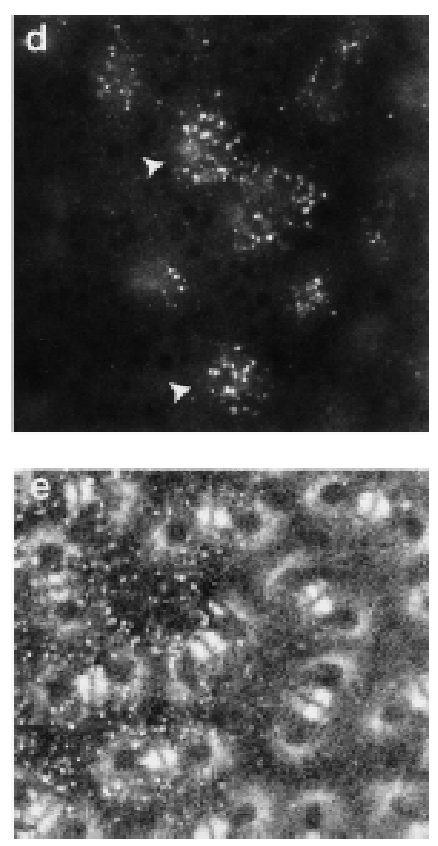

Figure 1. Staufen binds to the prospero $3^{\prime}$ UTR in vivo. Injection of the bcd 3' UTR into the precellularization embryo has been shown to recruit Staufen into ribonucleoprotein particles at the site of injection (Ferrandon et al. 1994). We tested whether the prospero 3' UTR also interacts with Staufen in this assay. In vitro-transcribed RN A was injected into living embryos expressing two different GFP fusion proteins: GFP-Staufen and Tau-GFP (Brand 1995; Micklem et al. 1997). (a) Full-length prospero 3' UTR RN A $(1.5 \mathrm{~kb})$ recruits Staufen to the site of injection within 5-10 min (arrowhead). (b) A similar result is obtained with a 650-nucleotide segment from the $3^{\prime}$ end of the prospero $3^{\prime}$ UTR (arrowhead). (c) Conversely, an 800-nucleotide fragment from the $5^{\prime}$ end of the prospero $3^{\prime}$ UTR recruits almost no Staufen to the site of injection, even after a 20-30 min incubation (arrowhead). (d) The ribonucleoprotein particles containing GFP-Staufen and the prospero 3' UTR cluster on the basal side of the nuclei and migrate with them as they move to the cortex of the embryo (arrowheads; 650-nucl eoti de fragment of the prospero 3' UTR was injected). (e) Although the RN P particles are distributed over the nuclei, there is no apparent association with astral microtubules (labeled with Tau-GFP). 


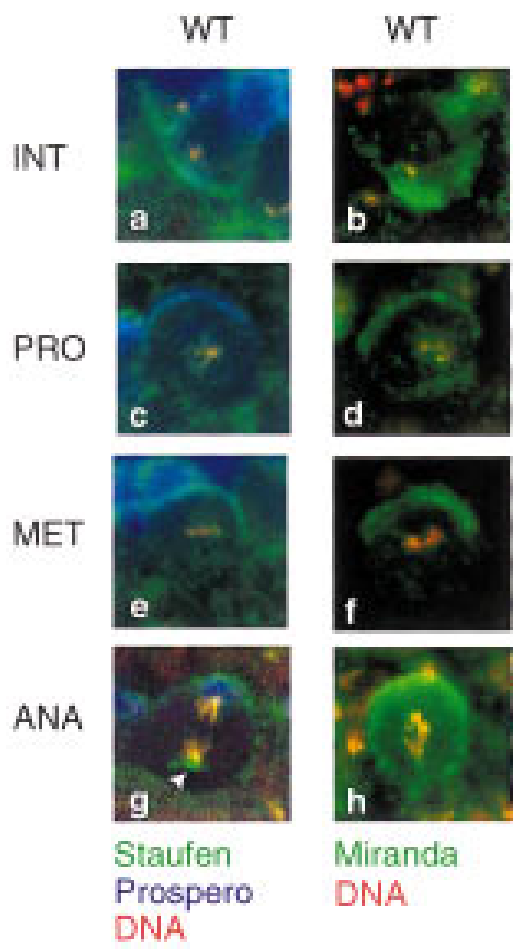

Figure 2. Staufen, Prospero, and Miranda colocalize throughout the cell cycle. Staufen (in green; $a, c, e, g$ ), Prospero (in blue; $a, c, e, g$ ), and M iranda (in green; b,d,f,h) colocalize in neuroblasts. At interphase, all three proteins are found on the apical side of the cell $(a, b)$; at prophase, they form a crescent at the basal cortex $(c, d)$, which is maintained at metaphase $(e, f)$ and anaphase $(g, h)$ before segregation into the GMC. Interestingly, Staufen is often also associated with the centrosome on the apical side of the neuroblast at anaphase (arrowhead in g), as is Miranda (see Fig. 5j). DNA is labeled in red in all panels; basal is up, apical down.

(Micklem 1998; C.M. Davidson and A.H. Brand, unpubl.).

To test whether either of these domains is required for Staufen localization in neuroblasts, we assayed the subcellular distribution of Staufen mutants that lack the dRBD 2 insert ( $\triangle \mathrm{dRBD} 2$ ) or the carboxy-terminal domain of Staufen ( $\triangle \mathrm{dRBD} 5)$. The removal of the dRBD2 insert has no effect on Staufen distribution in neuroblasts at any stage of the cell cycle (Fig. $3 a, c, e, g$ ). However, the loss of the carboxy-terminal 157 amino acids of Staufen eliminates both apical and basal localization. Staufen ${ }^{\Delta \mathrm{dRBD} 5}$ is distributed throughout the cytoplasm from interphase through mitosis (Figure $3 b, d, f, h)$. Therefore, the carboxyl terminus of Staufen is necessary to direct asymmetric distribution of the protein in neuroblasts. If the normal subcellular distribution of Staufen is mediated by a specific protein-protein interaction, then the site of the interaction may reside within the 157-amino-acid domain removed in Staufen ${ }^{\Delta \mathrm{dRBD} 5}$.

Staufen binds directly to Miranda through dRBD 5

Staufen dRBD5 does not interact with RNA in vitro (S.
Grunert and D. St Johnston, unpubl.) but is required in vivo for Staufen protein mRN A localization, suggesting that it may interact with other proteins that anchor Staufen at the apical and basal cortex, or mediate its transport from one side of the neuroblast to the other. To identify proteins that might interact with dRBD 5 to direct Staufen crescent formation, we carried out a yeast two-hybrid screen on a random-primed embryonic cDN A library using a LexA-dRBD 5 fusion protein as bait (Fields and Song 1989; Bartel et al. 1993; Poortinga et al. 1998). From 4,000,000 transformants, we isolated 10 positive clones, and were able to recover the library plasmid from 6 of these. All six clones contained the same insert, which encodes amino acids 506-776 of the Miranda protein.
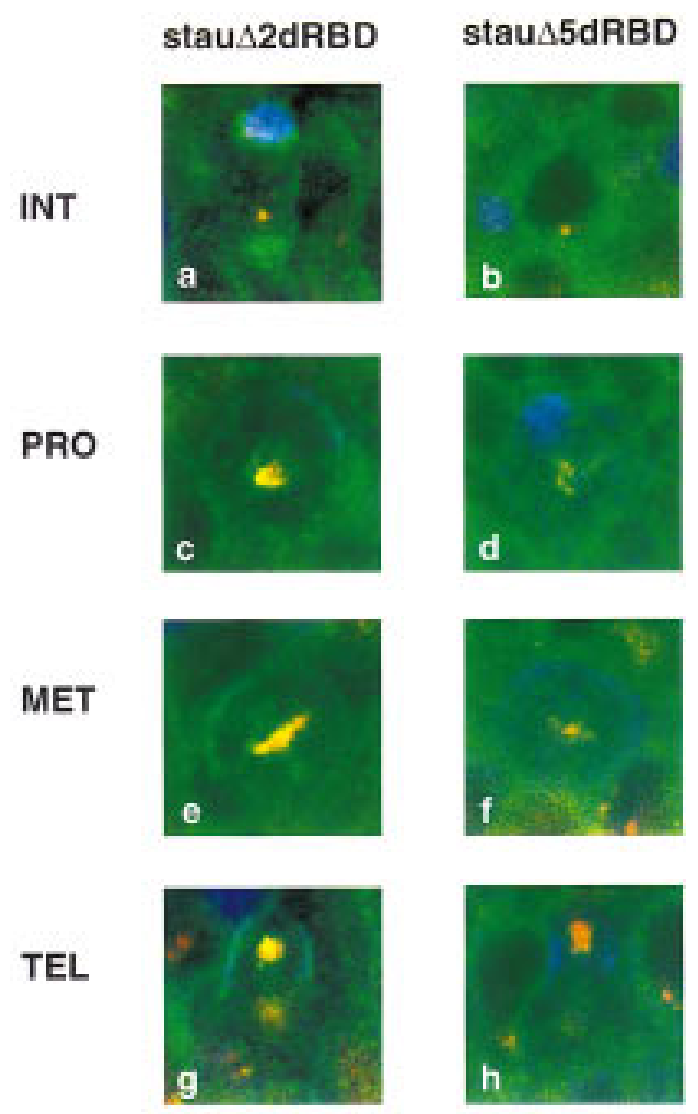

\section{Staufen \\ Prospero \\ DNA}

Figure 3. Deletion of dRBD5 eliminates Staufen localization. Deletion of the loop in dRBD2 of Staufen has little effect on protein localization throughout the cell cycle $(a, c, e, g)$. At interphase, Staufen ${ }^{\Delta \mathrm{dRBD} 2}$ concentrates on the apical side of the cell (a) before moving to the basal cortex during mitosis (c,e,g). Deletion of dRBD5, however, prevents Staufen localization to either the apical side of the neuroblast at interphase (b), or the basal side at prophase (d), metaphase (f), or anaphase (h). Staufen is labeled in green, Prospero in blue, and DNA in red in all panels; basal is up, apical down. 
To test the specificity of the dRBD5/M iranda interaction, we retransformed the $M$ iranda clone into yeast that contained either the original bait (lexA-dRBD5) or the control baits (lexA-lamin or lexA-BRCA2). Only yeast containing both lexA-dRBD 5 and M iranda-VP16 express high levels of $\beta$-gal actosidase (Fig. 4b). Furthermore, this region of $M$ iranda does not interact with all dRBDs, because no $\beta$-galactosidase activity is observed when $\mathrm{Mi}$ randa-VP16 is cotransformed with LexA-Staufen dRBD 1 (Fig. 4b). Thus, Miranda binds specifically to Staufen dRBD5, and does not interact with a closely related domain from the same protein.

To more precisely map the regi on of Miranda protein that interacts with Staufen-dRBD5, we divided the fragment of Miranda identified in the yeast two-hybrid screen into two parts (amino acids 506-638 and amino acids 639-776), and examined their interaction with dRBD5 in a GST pull-down assay (Hagemeier et al. 1991). ${ }^{35}$ S-Labeled Staufen-dRBD5 coprecipitates with both the full-length fragment, GST-Miranda amino acids 506-776, and the amino-terminal segment of this region, GST -M iranda amino acids 506-638, but shows no interaction above background with the carboxy-terminal segment, GST-M iranda amino acids 639-776 (Fig. 4c). This suggests that the Staufen binding site in Miranda corresponds to the predicted coiled-coil domain that extends from amino acids 526-593.
Miranda colocalizes with Staufen throughout the cell cycle

We have shown that Miranda and Staufen can interact in a yeast two-hybrid screen and in vitro. If the two proteins also interact in Drosophila embryos, they should colocalize in neuroblasts at specific stages of the cell cycle. Miranda has been reported to be evenly distributed throughout the cytoplasm and around the cell cortex at interphase, and to form a basal crescent during mitosis (Ikeshima-Kataoka et al. 1997; Shen et al. 1997). Therefore, Staufen might bind to one protein on the apical side of the neuroblast, for example, Inscuteable, and to a second on the basal side of the cell, Miranda. However, when we assay the subcellular distribution of Miranda we find that, like Staufen, Miranda concentrates predominantly on the apical side of the cell at interphase (Fig. 2b). Interestingly, miranda mRNA is also localized predominantly on the apical side of the neuroblast (Fig. 5m). Miranda protein then forms a crescent on the basal side of the neuroblast at prophase, where it remains until after cell division (Fig. 2d,f,h; IkeshimaKataoka et al. 1997; Shen et al. 1997). Therefore, the subcellular distribution of Miranda suggests that it might interact with Staufen at all stages of the cell cycle.

a

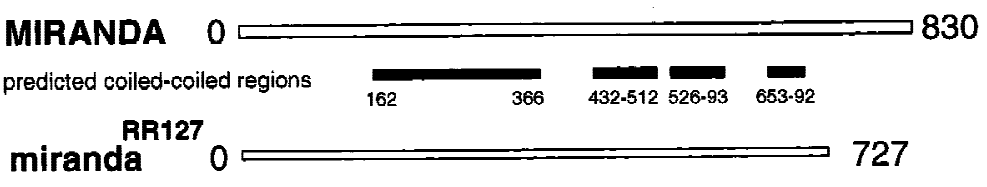
Bait used
Staufen dRBD5
Miranda Fragment Recovered

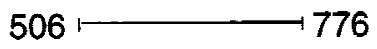

Prospero (820-1026)

(Shen et al.)

Prospero (1-1403)

(lkeshima-Kataoka et al.)

b

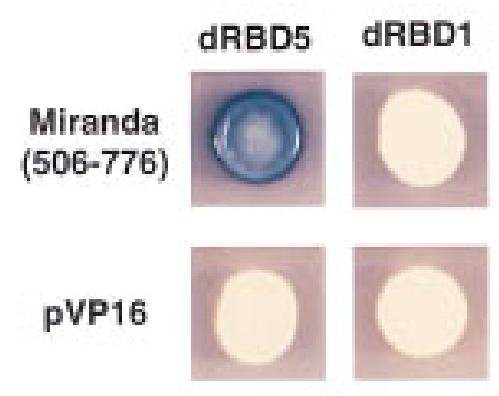

300 830

547 830

C

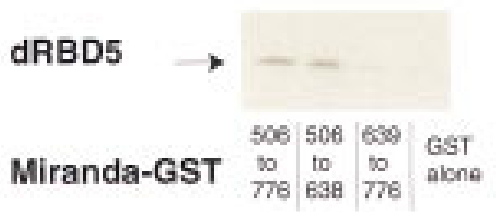

Figure 4. Staufen interacts with Miranda. (a) miranda encodes a protein of 830 amino acids (open rectangle in a, marked 0-830 amino acids), within which are four potential coiled-coil domains (solid boxes). The carboxy-terminal 103 amino acids are deleted in mirandaRR127 (open rectangle, 0-727 amino acids). By use of Staufen dRBD5 as bait in a yeast two-hybrid screen, we isolated six clones, all of which encode amino acids 506-776 of Miranda (black line). When amino acids 820-1026 of Prospero were used as bait in a similar screen, Shen et al. (1997) isolated amino acids 300-830 of Miranda. Ikeshima-Kataoka et al. (1997) used amino acids 11403 of Prospero as bait and recovered a fragment of Miranda encoded by amino acids 547-830. (b) Only yeast expressing both Staufen dRBD5 and Miranda amino acids 506-776 activate expression of lacZ (blue colony). Coexpression of either Staufen dRBD 1 with Miranda, Staufen dRBD 5 with the pVP16 vector, or Staufen dRBD1 with the pVP16 vector, does not activate transcription (white colonies). (c) ${ }^{35} \mathrm{~S}-$ Label ed Staufen dRBD 5 interacts in vitro with GST Miranda amino acids 506-776 (first lane) and GST Miranda amino acids 506-638 (second lane) but not with GST -M iranda amino aci ds 639-776 (third lane) or GST alone (fourth lane). 


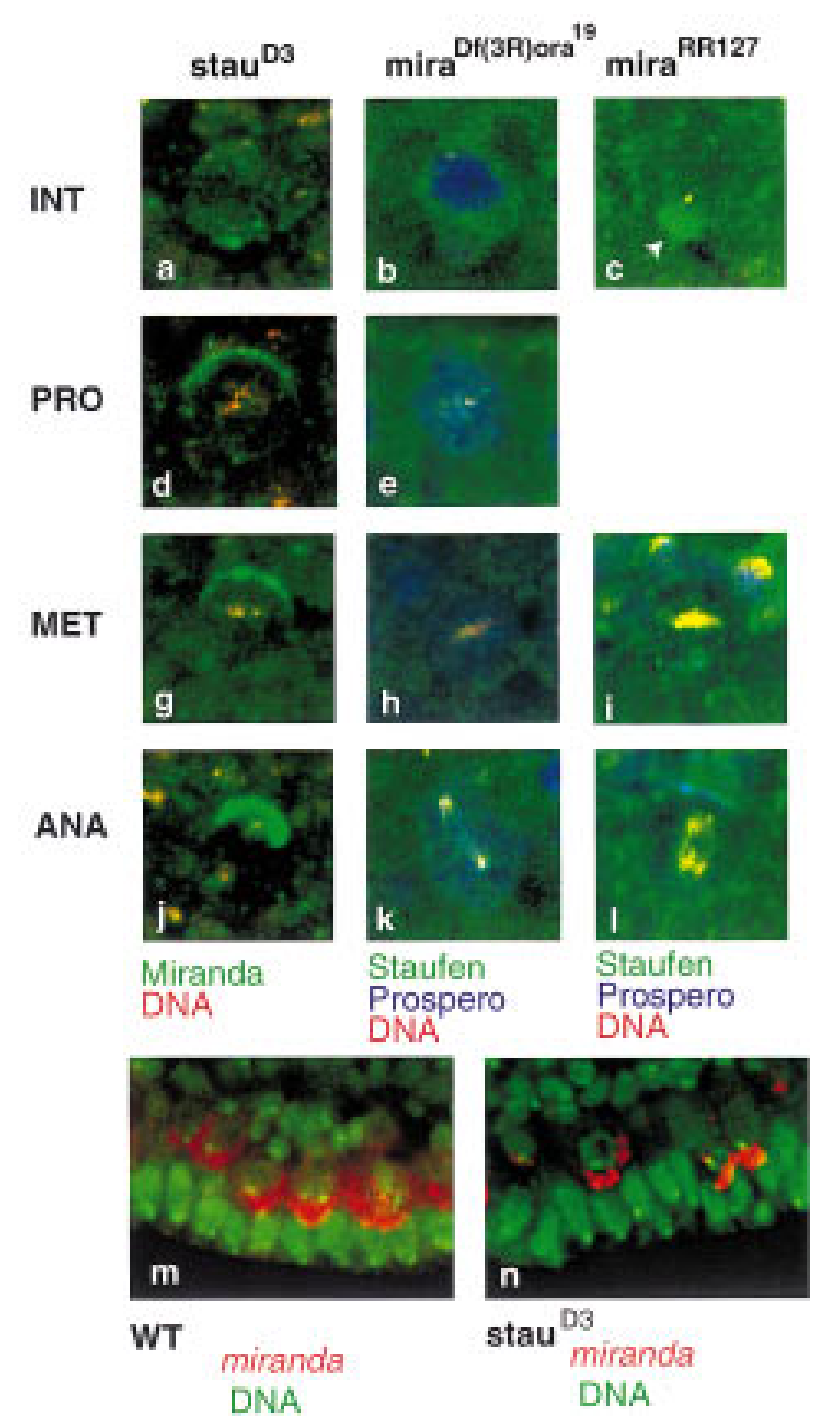

Figure 5. The subcellular localization of Staufen requires $\mathrm{Mi}$ randa. Miranda localizes normally throughout the cell cycle in staufen ${ }^{\mathrm{B}}$ mutants $(a, d, g, j$; Miranda labeled in green, DN A in red). Staufen subcellular local ization, however, is absolutely dependent on Miranda (b,e,h,k; Staufen label ed in green, Prospero in blue, DN A in red). In mirandaRR127, Staufen is appropriately localized, but concentrates more strongly at the apical side of the neuroblast at interphase (arrowhead in c; Staufen label ed in green, Prospero in blue, DN A in red), and forms a weaker basal crescent at mitosis (i,I). miranda mRN A (labeled in red, DNA in green) localizes predominantly on the apical side of the neuroblast throughout the cell cycle in both wild-type $(\mathrm{m})$ and staufen ${ }^{\mathrm{3}}$ embryos (n).

The subcellular distribution of Staufen requires Miranda

Miranda interacts with both Prospero and $\mathrm{Numb}$ in vitro, but is required to tether only Prospero to the cell membrane in vivo (Ikeshima-Kataoka et al . 1997; Shen et al. 1997). $\mathrm{N}$ umb is itself a membrane-associated protein and although it may form a complex with Miranda, the interaction is not necessary to maintain $\mathrm{Numb}$ at the cortex. Prospero, in contrast, is a homeodomain protein that moves into the nucleus unl ess tethered by M iranda at the membrane. Staufen is not itself membrane associated, suggesting that it too may be tethered to the cortex by a specific protein-protein interaction. As Staufen binds to Miranda in a yeast two-hybrid screen, and the two proteins col ocal ize in neuroblasts, it seemed possible that Miranda might anchor Staufen to the cortex.

To test if Miranda is required to localize Staufen in vivo, we followed the subcellular distribution of Staufen in miranda null mutant embryos [Df(3R)ora $\left.{ }^{19}\right]$. In the absence of Miranda, Staufen is found throughout the neuroblast cytoplasm at all stages of the cell cycle (Fig. 5b,e,h,k). Similar results are seen in an EMSinduced al lele, mirandaYY227 (data not shown). Prospero protein is also evenly distributed (Fig. 5b,e,h,k). We assayed the distribution of Miranda in a staufen mutant, to determine whether the localization of the two proteins is mutually dependent (Fig. 5a,d,g,j). Miranda localizes normally in the absence of Staufen, suggesting that it acts upstream of both Staufen and Prospero in the process of asymmetric protein localization. miranda mRNA also remains apical in staufen mutant embryos (Fig. 3n).

Although Prospero is never localized at the cortex in most mi randa mutants, one mutant, mi randa ${ }^{\mathrm{RR} 127}$, does localize Prospero but fails to release it from the cortex even after the two proteins are partitioned to the GMC (Ikeshima-Kataoka et al. 1997). The mutation removes the carboxy-terminal 103 amino acids of Miranda, within which lie sites for phosphorylation by protein kinase $C$ (PKC). If phosphorylation regulates the release of Prospero from Miranda, then the Staufen-M iranda interaction might be similarly regulated.

We assayed the subcellular distribution of Staufen in mirandaRR127 mutant embryos. In these embryos, Prospero remains at the cortex and cannot enter the GMC nucleus. We cannot determine whether Staufen localization in the GMC is affected, because Staufen is normally cytoplasmic or cortical, but we do observe an increased accumulation of Staufen at the apical side of the neuroblast at interphase (Fig. 5c). At metaphase, less Staufen is concentrated in a basal crescent (cf. Fig. $5 i$ with Fig. 2e). Therefore, Miranda may anchor Staufen at the apical side of the cell, and regulate the release of both proteins to allow formation of a basal crescent at prophase.

\section{prospero mRNA localization requires Miranda}

We have shown that Miranda mediates both the apical and basal localization of Staufen and may regulate its transition from apical to basal during the cell cycle. If prospero mRNA localization is Staufen dependent then, by extension, prospero mRN A should be mislocalized in the absence of M iranda. As predicted, prospero mRN A is present throughout the cytoplasm in miranda mutant embryos (Fig. 6c-e). Miranda, therefore, mediates the asymmetric distribution not only of the Prospero protein, but also of its mRNA. 


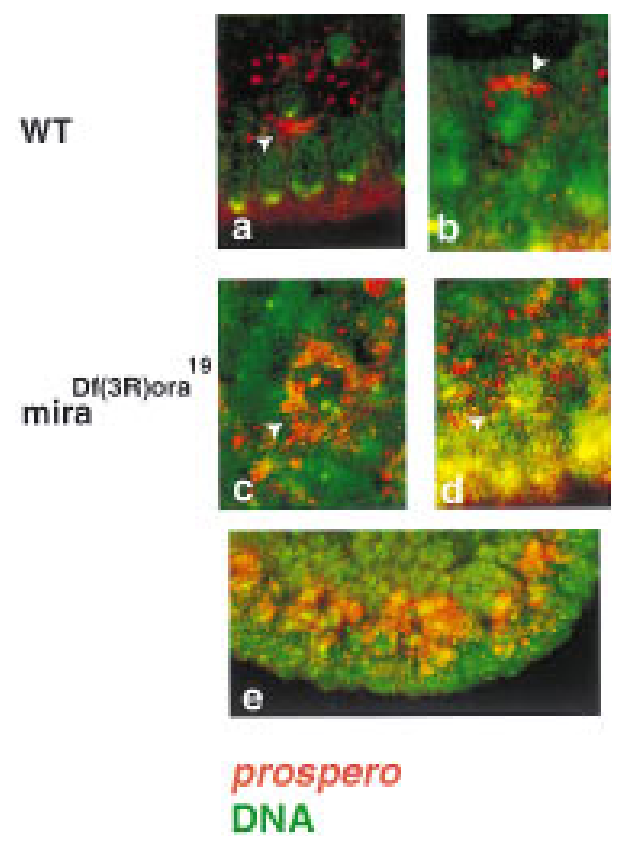

Figure 6. Miranda is required to localize prospero mRNA. prospero mRNA localizes on the apical side of neuroblasts at interphase (arrow in a) and moves to the basal cortex during mitosis (arrowhead in b). In the absence of M iranda, the RN A is evenly distributed in all neuroblasts (c,d,e). The arrowheads in $c$ and $d$ indicate individual neuroblasts. prospero mRNA is labeled in red, DNA in green.

\section{Discussion}

Miranda colocalizes with Staufen and prospero mRNA throughout the cell cycle

Here we demonstrate that the subcellular distribution of Miranda and Staufen is sufficient to explain all phases of prospero mRNA localization in neuroblasts. First, Staufen interacts in vivo with the prospero mRNA through a 650-nucleotide region of the $3^{\prime}$ UTR. Second, Staufen colocalizes with prospero mRNA in neuroblasts throughout the cell cycle. Staufen first concentrates on the apical side of the neuroblast at interphase, then moves to the basal side of the cell at prophase, and segregates preferentially into the GMC at cytokinesis. Third, both the apical and basal localization of Staufen are eliminated when dRBD5 is deleted. This region is sufficient to bind to Miranda in a yeast two-hybrid screen and in a GST pull-down assay. Fourth, we show that Miranda colocalizes with Staufen protein and prospero mRN A throughout cell cycle, concentrating first on the apical side of the cell and then forming a basal crescent at prophase. Finally, M iranda is required in vivo to localize Staufen, both apically and basally, and for prospero mRNA localization. Thus, Miranda, which localizes Prospero protein, also localizes prospero mRNA through its interaction with the RNA binding protein, Staufen (Fig. 7).

\section{Miranda and Staufen interact in vivo}

If RNA localization is an integral part of the process of asymmetric protein segregation, then something must coordinate the two processes. Here we show that Miranda performs this function. Miranda is a novel protein containing four coiled-coil structures, two leucine zippers, four putative destruction boxes, and eight potential PKC phosphorylation sites (Ikeshima-Kataoka et al. 1997; Shen et al. 1997). The carboxy-terminal 103 amino acids, which encode the PKC phosphorylation sites and are removed in miranda ${ }^{\mathrm{RR} 127}$, regulate the release of Prospero from the cortex. The cell cycle control of Prospero release, and Miranda turnover, may, therefore, be regulated by phosphorylation.

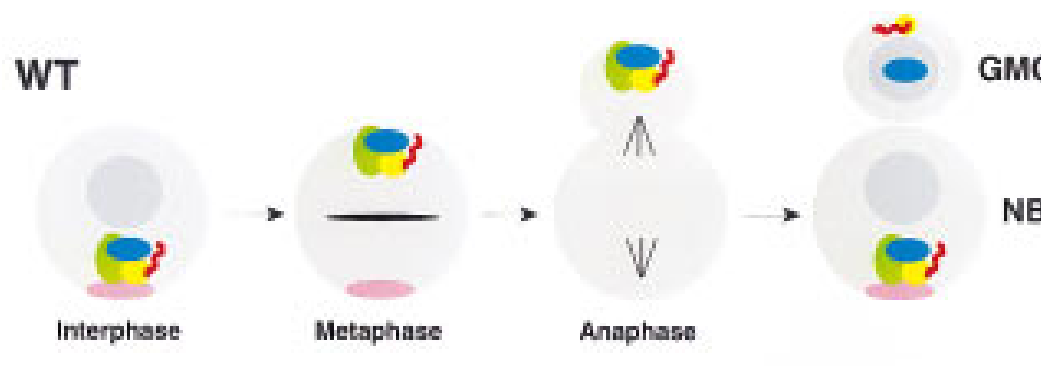

Figure 7. Miranda mediates the asymmetric segregation of Prospero, Staufen, and prospero mRN A. (Top) In wild-type neuroblasts, Miranda forms a complex with Prospero protein, Staufen and prospero mRNA, on the apical side of the cell at interphase. The complex moves to the basal side of the cell during mitosis, where it is anchored at the cortex prior to segregation into the GMC. Miranda is then degraded and Prospero enters the GMC nucleus. (Bottom) In the absence of Miranda, Staufen and prospero mRNA are randomly distributed in the neuroblast cytoplasm at interphase, whereas Prospero enters the nucleus. During mitosis, all three factors are evenly partitioned between the neurobl ast and the budding GMC. After cytokinesis, Prospero enters the nucleus of both the neuroblast and the GMC, whereas Staufen and prospero mRNA remain randomly distributed in the cytoplasm of both cells. 
We show that Staufen binds to amino acids 506-638 of Miranda, and that Staufen is still localized in miran$\mathrm{da}^{\mathrm{RR} 127}$ mutants. The Staufen binding site is contained within a domain of $\mathrm{M}$ iranda that has been shown also to interact with Prospero (amino acids 445-727; IkeshimaKataoka et al. 1997). This region encodes the third and fourth coiled-coil domains (Fig. 4a). Staufen binds to the 132 amino acids between 506-638, which encode the third coiled-coil domain.

Staufen dRBD5 is an unusual example of the dRBD protein motif, which contains the conserved amino acids that form the hydrophobic core of the domain, but lacks the basic amino acids required for RNA binding (St Johnston et al. 1992; Bycroft et al. 1995). Because dRBD5 does not bind dsRN A in vitro (S. Grunert, D.R. Micklem, and D. St Johnston, unpubl.), its conservation suggests that it might be involved in some other aspect of Staufen function. We show that the carboxy-terminal 157 amino acids of Staufen mediate its interaction with Miranda. The removal of this domain, in Staufen ${ }^{\Delta d R B D 5}$, eliminates all aspects of Staufen subcellular localization. The only conserved region within this 157 amino acids is dRBD5. The 78 amino acids encompassing dRBD5 are sufficient to bind to Miranda in a yeast two-hybrid screen and in vitro, further defining the interaction domain.

Miranda coordinates the asymmetric distribution of Prospero and its mRNA

Miranda has been shown to anchor Prospero protein to the basal cortex of the neuroblast, and to regulate its release from the cortex once segregated to the GMC. Here we demonstrate that $\mathrm{M}$ iranda al so di rects the subcellular distribution of Staufen and, in so doing, local izes the prospero mRN A. In the absence of Miranda, Prospero enters the neuroblast nucleus, and Staufen and prospero mRN A are evenly distributed in the cytoplasm.

Miranda binds to Prospero protein and to Staufen, which in turn binds prospero mRNA, to form a complex on the apical side of the neuroblast. The complex may be anchored by Inscuteable at interphase, and then released as the cell cycle progresses. In mirandaRR127, Staufen accumulates on the apical side of the cell, suggesting that Miranda may regulate release from the apical cortex. Miranda, Prospero, Staufen, and prospero mRNA then move as a group to the basal side of the cell during mitosis, a process that appears to require actin microfilaments. Staufen and M iranda al so associate with the apical centrosome, although the importance of this interaction is unclear. Once at the basal cortex, the complex is anchored by factors that have not, as yet, been identified. However, as $M$ iranda acts as the adapter between protei $n$ and RNA localization, these factors may be isolated in screens for other $\mathrm{M}$ iranda binding proteins.

After cytokinesis, Miranda is rapidly degraded in the GMC, and Prospero is rel eased and enters the nucleus. It may be important, therefore, to minimize translation of new Miranda protein in the GMC. Whereas prospero mRNA is specifically segregated to the GMC, miranda
mRN A remains tightly anchored on the apical si de of the neuroblast. By tethering miranda mRNA in this way, $M$ iranda protein, but not miranda $m R N A$, is partitioned to the GMC at cell division.

Several interesting questions remain to be answered. What regulates the release of Miranda from the apical side of the cell? How are M iranda, Prospero, Staufen, and prospero mRN A transported to the basal si de of the neuroblast? Do they move as a complex, and how are they anchored at the basal cortex? Prospero and Staufen bind to the same region of Miranda, but it is not known whether they bind to the same molecule simultaneously. The answers to these questions may help to elucidate the mechanism of asymmetric protein and RNA localization not only in the nervous system, but also in other tissues, and in other organisms.

\section{Materials and methods}

Drosophila mutants and transgenic lines

Flies carrying deficiency Df(3R)ora ${ }^{19}$, which deletes miranda (Shen et al. 1997), were a kind gift of Yuh N ung Jan (U niversity of California, San Francisco). The mirandaRR127 and mirandaYY227 mutants (Ikeshima-Kataoka et al. 1997) were kindly provided by Fumio $\mathrm{M}$ atsuzaki ( $\mathrm{N}$ ational Institute of $\mathrm{N}$ euroscience, Tokyo, Japan). staufen ${ }^{\mathrm{D} 3}$ is a protein null (St Johnston et al. 1991).

Expression of Myc-tagged derivatives of Staufen was driven in the germ line of homozygous staufen ${ }^{\mathrm{D} 3}$ females from the $\alpha 4$ tubulin promoter. The Staufen fusion proteins consist of the first 9 amino acids of $\alpha 4$ tubulin, a 16-amino-acid myc epitope, followed by amino acids 18-1026 of Staufen. To express wildtype myc-tagged Staufen, an Fspl-Clal fragment (324-1140, numbered as in GenBank M 69111) from staufen cDNA E3 (St Johnston et al. 1991) was cloned into the BamHI site of D277 (a modified pCaTub67MatpolyA vector; Micklem et al. 1997) to generate D287. The CellI-N otl fragment of CDNA E3 (483-end) was then inserted between the Celll site and the N otl site in the polylinker to produce D288.

The Staufen ${ }^{\Delta \mathrm{dRBD} 5}$ derivative was generated in a similar fashion, but lacks the carboxy-terminal 157 amino acids of Staufen, downstream of the Pvull site at position 2879. Staufen ${ }^{\Delta \mathrm{dRBD} 2}$ is identical to D288, except that the 99-amino-acid insertion in the middle of dRBD2 has been replaced with the 8-amino-acid loop that occurs in the equivalent position in dRBD3. Further information on the sequence of these constructs is available on request. DN A constructs were introduced into the germ line of w; stau ${ }^{\mathrm{D} 3}$, sp/CyO flies by P-element-mediated transfomation (Rubin and Spradling 1982). Multiple transformant lines were obtained for each construct and lines giving the highest levels of expression were analyzed further.

Expression of GFP-Staufen was driven from the $\alpha 4$ tubulin promoter in vector D277M. We used the GFP variant, mGFP6, to enhance the fluorescence and solubility of the fusion protein. Staufen fusions with other GFP variants give no fluorescence, possibly because of the production of an insoluble fusion protein [data not shown; see Siemering et al. (1996) for a discussion of solubility and fluorescence]. mGFP6 carries mutations that improve the maturation and spectral properties of the protein, F64L, S65T (Heim et al. 1995; Cormack et al. 1996) and V163A, I167T, S175G (Heim et al. 1994; Siemering et al. 1996), and codon usage changes that remove a cryptic intron and optimize expression (Hasel off et al. 1997; data not shown). The sequence 
of mGFP6 is available on request. mGFP6 was PCR amplified as a Pstl-Bgl II fragment and cl oned into D277M , replacing the myc epitope tag. Staufen was then cloned as a BgllI-Notl fragment from D288 downstream of mGFP6. The transgenic line used in this study, $\alpha 4$ tubulin-mGP6-Staufen ${ }^{2.1}$, is an insert on 3R that rescues the maternal effect lethal ity of a staufen null mutation.

\section{RNA injection}

prospero RNA was transcribed with an Ambion transcription kit. RN A was injected at a concentration of $1 \mu \mathrm{g} / \mathrm{ml}$ (in water) into 0-1 hr embryos (Ferrandon et al. 1994) expressing a GFPStaufen fusion protein, driven by the $\alpha 4$ tubulin promoter (described above), and a Tau-GFP fusion protein (Brand 1995), also driven by the $\alpha 4$ tubulin promoter (Micklem et al. 1997). Time lapse images of injected embryos were collected by confocal microscopy with a BioRad M RC1024 and a N ikon E800 microscope. Images were imported into Adobe Photoshop 4.0, and assembled in Adobe Illustrator 6.0.

\section{Immunohistochemistry}

Antibody staining was carried out according to Patel (1994) with the following modifications: To preserve the cytoskel eton, embryos were fixed for $5 \mathrm{~min}$ in a 1:1 mix of undiluted $(37 \%)$ formal dehyde and heptane. PBS, $0.1 \%$ Triton X-100 replaces PEM (100 mm PIPES, $2 \mathrm{~mm}$ EGTA, 1 mM $\mathrm{MgSO}_{4}$ ) throughout. Rabbit anti-Staufen (St Johnston et al. 1991) was used at a dilution of 1:500; mouse anti-Prospero mAb M R2A (Spana and Doe 1995; a kind gift from Chris Doe) was used at a dilution of 1:2; rabbit anti-M iranda A 96c (Shen et al. 1997; a kind gift from Y uh N ung Jan) was used at 1:1000. Secondary antibodies, directly conjugated to FITC, Texas Red, or Cy5 were used at a dilution of 1:200. Embryos were mounted in DNA stain solution (Lundell and Hirsh 1994; sonicated $2 \mathrm{mg} / \mathrm{ml}$ of 1,4-phenylenediamine in $4 \mathrm{mM} \mathrm{N}_{2} \mathrm{CO}_{3}, 90 \%$ glycerol) and visualized by confocal microscopy, as above.

\section{In situ hybridization}

A 1.5-kb BamHI fragment of the prospero CDNA (a kind gift from W. Chia, Institute of M olecular and Cell Biology, N ational University of Singapore) was used as a template to generate a random primed, digoxigenin-labeled, DNA probe (Boehringer $M$ annheim). In situ hybridization to whole mount embryos was carried out as described previously (Tautz and Pfeifle 1989), with embryos incubated overnight in a hybridization mix at $48^{\circ} \mathrm{C}$. The embryos were washed, then incubated with alkaline phosphatase-conjugated anti-digoxigenin antibodies. The alkaline phosphatase reaction was carried out by use of the fluorescent substrate, HNPP/Fast Red TR (Boehringer Mannheim) as described by Goto and Hayashi (1997). Embryos were mounted in DNA stain solution and visualized by confocal microscopy, as above.

\section{Yeast two-hybrid screen and GST pull downs}

To construct the LexA-dRBD 5 bait, the carboxy-terminal 78 amino acids of Staufen (bases 3116-3355) were amplified by the primers GGAATTCGCTGGAGTGCACATGAAGGAGCA and CGGGATCCTTACCCCAGCTTGCTGAGGAT, and cloned between the EcoRI and BamHI sites of PBTM 116-nIs (Bartel et al. 1993). The resulting plasmid was transformed into yeast strain L40 (Hollenberg et al. 1995). The same yeast were then transformed with a random-primed cDNA library in pVP16 made from 0- to 4-hr embryonic mRN A (a generous gift of Gretchen Poortinga and Susan Parkhurst; Poortinga et al. 1998). Of 4,000,000 transformants, 138 colonies grew on $\mathrm{His}^{-}$ plates. Ten of these $\mathrm{His}^{+}$colonies also showed $\beta$-galactosidase activity. cDNA library plasmids were successfully recovered from six of these. The specificity of the interaction between these positive clones and lexA-dRBD 5 was examined further by testing for their ability to activate his 3 and lacZ expression when cotransformed with the original bait. LexA-Lamin, LexABRCA2, and LexA-Staufen dRBD 1 served as negative controls.

For GST pull down assays, three fragments of $\mathrm{M}$ iranda (amino acids 506-776, amino acids 506-638, or amino acids 639-776) were cloned in-frame with GST in pGEX2T (Pharmacia), and purified after expression in Escherichia coli C41 (Bannister and Kouzarides 1996; Miroux and Walker 1996). Staufen dRBD5 (amino acids 937-1026) was cloned into plN G 14 (Taunton et al. 1996; Brehm et al. 1998). In vitro translated [35S]methioninedRBD 5 was used in GST pull down assays, as described by Hagemeier et al. (1991). Briefly, 500 ng of GST fusion protein on beads was incubated with $2-5 \mathrm{ml}$ of dRBD5 in Z buffer. The beads were then washed three times in NETN buffer and bound protein was resolved by SDS-PAGE.

\section{Acknowledgments}

We thank Fumio M atsuzaki for communicating results prior to publication. We are grateful to Gretchen Poortinga and Susan Parkhurst for kindly providing the yeast two-hybrid library prior to publication. For generously providing DN A constructs, antibodies, and Drosophila lines, we thank Bill Chia, Chris Doe, Yuh Nung Jan, and Fumio Matsuzaki. We thank Chris Phelps for comments on the manuscript. A.J.S. is supported by a BBSRC special studentship; J.A. is funded by a studentship from the Boehringer Ingel heim Fonds; J.H. is supported by a grant from the Biotechnology and Biological Sciences Research Council (BBSRC). This work was funded by the Well come Trust.

The publication costs of this article were defrayed in part by payment of page charges. This article must therefore be hereby marked "advertisement" in accordance with 18 USC section 1734 solely to indicate this fact.

\section{References}

Bannister, A.J. and T. Kouzarides. 1996. The CBP co-activator is a histone acetyltransferase. Nature 384: 641-643.

Bartel, P.L., C. Chien, R. Sternglanz, and S. Fields. 1993. Using the two hybrid system to detect protein-protein interactions. In Cellular interactions in development: A practical approach (ed. D.A. Hartley), pp. 153-179. IRL Press, Oxford, UK.

Bobola, N., R.-P. Jansen, T.H. Shin, and K. N asmyth. 1996. Asymmetric accumulation of Ashlp in postanaphase nuclei depends on a myosin and restricts yeast mating-type switching to mother cells. Cell 84: 699-709.

Brand, A. 1995. GFP in Drosophila. Trends Genet.11: 324-325.

Brehm, A., E.A. Miska, D.J. McCance, J.L. Reid, A. Bannister, and T. Kouzarides. 1998. Retinoblastoma protein recruits histone deacetylase to repress transcription. Nature 391: 597-601.

Broadus, J. and C.Q. Doe. 1997. Extrinsic cues, intrinsic cues and microfilaments regulate asymmetric localization in Drosophila neuroblasts. Curr. Biol. 7: 827-835.

Broadus, J., S. Fuerstenberg, and C.Q. Doe. 1998. Staufen-dependent localization of prospero mRNA contributes to neuroblast daughter-cell fate. Nature 391: 792-795.

Bycroft, M., S. Grünert, A.G. Murzin, M. Proctor, and D. St 
Johnston. 1995. N M R solution structure of a dsRN A binding domain from Drosophila Staufen protein reveals homology to the $\mathrm{N}$ terminal domain of ribosomal protein S5. EMBO J. 14: 3563-3571.

Chenn, A. and S.K. M cConnell. 1995. Cleavage orientation and the asymmetric inheritance of $\mathrm{N}$ otch 1 immunoreactivity in mammalian neurogenesis. Cell 82: 631-641.

Clark, I., E. Giniger, H. Ruohola-Baker, L.Y. Jan, and Y.N. Jan. 1994. Transient posterior localization of a kinesin fusion protein reflects anteroposterior polarity of the Drosophila oocyte. Curr. Biol. 4: 289-300.

Cormack, B.P., R.H. Valdivia, and S. Falkow. 1996. Facs optimized mutants of the green fluorescent protein (GFP). Gene 173: 33-38.

Doe, C.Q., Q. Chu-LaGraff, D.M. Wright, and M .P. Scott. 1991. The prospero gene specifies cell fates in the Drosophila central nervous system. Cell 65: 451-465.

Ephrussi, A., L.K. Dickinson, and R. Lehmann. 1991. oskar organizes the germ plasm and directs localization of the posterior determinant nanos. Cell 66: 37-50.

Ferrandon, D., L. El phick, C. N üsslein-Volhard, and D. St Johnston. 1994. Staufen protein associates with the $3^{\prime}$ UTR of bicoid mRN A to form particles that move in a microtubuledependent manner. Cell 79: 1221-1232.

Fields, S. and O. Song. 1989. A novel genetic system to detect protein-protein interactions. Nature 340: 245-246.

Goto, S. and S. Hayashi. 1997. Cell migration within the embryonic limb primordium of Drosophila as revealed by a novel fluorescence method to visualize mRNA and protein. Dev. Genes Evol. 207: 194-198.

Hagemeier, C., A. Cook, and T. Kouzarides. 1991. The retinoblastoma protein binds E2F residues required for activation in vivo and TBP binding in vitro. Nucleic Acid Res. 21: 160162.

Hasel off, J., D.R. Siemering, D.C. Prasher, and S. Hodge. 1997. Removal of a cryptic intron and subcellular localization of green fluorescent protein are required to mark transgenic Arabidopsis plants brightly. Proc. Natl. Acad. Sci. 94: 21222127.

Heim, R., D.C. Prasher, and R.Y. Tsien. 1994. Wavel ength mutations and post-translational autoxidation of green fluorescent protein. Proc. Natl. Acad. Sci. 91: 12501-12504.

Heim, R., A.B. Cubitt, and R.Y. Tsien. 1995. Improved green fluorescence. Nature 373: 663-664.

Hirata, J., H. N akagoshi, Y. N abeshima, and F. M atsuzaki. 1995. Asymmetric segregation of the homeodomain protein Prospero during Drosophila devel opment. Nature 377: 627-630.

Hollenberg, S.M., R. Sterngl anz, P.F. Cheng, and H. Weintraub. 1995. Identification of a new family of tissue specific basic helix-loop-helix proteins with a two hybrid system. Mol. Cell Biol. 15: 3813-3822.

Ikeshima-Kataoka, H., J.B. Skeath, Y. N abeshima, C.Q. Doe, and F. M atsuzaki. 1997. M iranda directs Prospero to a daughter cell during Drosophila asymmetric divisions. Nature 390: 625-629.

Kim-Ha, J., J.L. Smith, and P.M. M acdonald. 1991. oskar mRN A is localized to the posterior pole of the Drosophila oocyte. Cell 66: 23-35.

Kim-Ha, J., K. Kerr, and P.M. M acdonald. 1995. Translational regulation of oskar messenger RNA by Bruno, an ovarian RN A binding protein, is essential. Cell 81: 403-412.

Knoblich, J.A., L.Y. Jan, and Y.N . Jan. 1995. Asymmetric segregation of $\mathrm{Numb}$ and Prospero during cell division. Nature 377: 624-630.

Kraut, R. and J.A. Campos-Ortega. 1996. inscuteable, a neural precursor gene of Drosophila, encodes a candidate for a cy- toskeletal adapter protein. Dev. Biol. 174: 65-81.

Kraut, R., W. Chia, L.Y. Jan, Y.N. Jan, and J.A. Knoblich. 1996. Role of inscuteable in orienting asymmetric cell divisions in Drosophila. Nature 383: 50-55.

Li, P., X.H. Yang, M. Wasser, Y. Cai, and W. Chia. 1997. Inscuteable and staufen mediate asymmetric localization and seg regation of prospero RN A during Drosophila neuroblast cell divisions. Cell 90: 437-447.

Long, R.M., R.H. Singer, X. M eng, I. Gonzalez, K. N asmyth, and R.-P. Jansen. 1997. M ating type switching in yeast controlled by asymmetric localization of ASH1 mRNA. Science 277: 383-387.

Lundell, M.J. and J. Hirsh. 1994. A new visible light DN A fluorochrome for confocal microscopy. BioTechniques 16: 434440.

Matsuzaki, F., K. Koisumi, C. Hama, T. Yoshioka, and T. Nabeshima. 1992. Cloning of the Drosophila prospero gene and its expression in ganglion mother cells. Biochem. Biophys. Res. Comm. 182: 1326-1332.

Micklem, D.R. 1998. "T rans-acting factors required for the posterior localisation of oskar mRNA." Ph.D. thesis, Cambridge University, Cambridge, UK.

Micklem, D.R., R. Dasgupta, H. Elliott, F. Gergely, C. Davidson, A. Brand, A. Gonzalez-Reyes, and D. St Johnston. 1997. mago nashi is required for the polarisation of the oocyte and the formation of perpendicular axes in Drosophila. Curr. Biol. 7: 468-478.

Miroux, B. and J.E. Walker. 1996. Over-production of proteins in Escherichia coli-mutant hosts that allow synthesis of some membrane proteins and globular proteins at high levels. J. Mol. Biol. 260: 289-298.

N elson, W.J. and K.K. Grindstaff. 1997. Cell polarity: Par for the polar course. Curr. Biol. 7: R562-R564.

Patel, N.H. 1994. Imaging neuronal subsets and other cell types in whole mount Drosophila embryos and larvae using antibody probes. In Drosophila melanogaster: Practical uses in cell and molecular biology (ed. L.S.B. Goldstein and E.A. Fyrberg), pp. 445-487. Academic Press, San Diego, CA.

Pokrywka, N .J. and E.C. Stephenson. 1991. Microtubules mediate the localization of bicoid RN A during Drosophila oogenesis. Development 113: 55-66.

- - - 1995. Microtubules are a general component of mRN A localization systems in Drosophila oocytes. Dev. Biol. 167: 363-370.

Poortinga, G., M. Watanabe, and S.M. Parkhurst. 1998. Drosophila CtBP: A Hairy-interacting protein required for embryonic segmentation and Hairy-mediated transcriptional repression. EMBO J. (in press).

Rhyu, M.S., L.Y. Jan, and Y.N. Jan. 1994. Asymmetric distribution of $\mathrm{Numb}$ protein during division of the sensory organ precursor cell confers distinct fates to daughter cells. Cell 76: 477-491.

Rubin, G.M. and A.C. Spradling. 1982. Genetic transformation of Drosophila with transposable elements vectors. Science 218: 348-353.

Schüpbach, T. and E. Wieschaus. 1989. Female sterile mutations on the second chromosome of Drosophila melanogaster. I. Maternal effect mutations. Genetics 121: 101-117.

Shen, C.P., L.Y. Jan, and Y.N . Jan. 1997. M iranda is required for the asymmetric localization of Prospero during mitosis in Drosophila. Cell 90: 449-458.

Siemering, K.R., R. Golbik, R. Sever, and J. Hasel off. 1996. Mutations that suppress the thermosensitivity of green fluorescent protein. Curr. Biol. 6: 1653-1663.

Sil, A. and I. Herskowitz. 1996. Identification of an asymmetrically local ized determinant, Ash1p, required for lineage-spe- 
cific transcription of the yeast $\mathrm{HO}$ gene. Cell 84: 711-722.

Spana, E.P. and C.Q. Doe. 1995. The Prospero transcription factor is asymmetrically localized to the cell cortex during neuroblast mitosis in Drosophila. Development 121: 31873195.

- - 1996. N umb antagonizes $\mathrm{N}$ otch signal ing to specify sibling neuron cell fates. Neuron 17: 21-26.

Spana, E., C. Kopczynski, C.S. Goodman, and C.Q. Doe. 1995. Asymmetric localization of numb autonomously determines sibling neuron identity in the Drosophila CNS. Development 121: 3489-3494.

St Johnston, D. 1995. The intracellular localization of messenger RN As. Cell 81: 161-170.

St Johnston, D., D. Beuchle, and C. N üsslein-Volhard. 1991. staufen, a gene required to localize maternal RNAs in the Drosophila egg. Cell 66: 51-63.

St Johnston, D., N.H. Brown, J.G. Gall, and M. Jantsch. 1992. A conserved double-stranded RNA-binding domain. Proc. Natl. Acad. Sci. 89: 10979-10983.

Takizawa, P.A., A. Sil, J.R. Swedlow, I. Herskowitz, and R.D. Vale. 1997. Actin-dependent localization of an RN A encoding a cell-fate determinant in yeast. Nature 389: 90-93.

Taunton, J., C.A. Hassig, and S.L. Schreiber. 1996. A mammalian histone deacetylase rel ated to yeast transcriptional regulator Rpd3p. Science 272: 408-411.

Tautz, D. and C. Pfeifle. 1989. In situ hybridization to embryos with non-radioactive probes. In Drosophila, a laboratory manual (ed. M. A shburner), pp. 194-198. Cold Spring Harbor Laboratory Press, Cold Spring Harbor, NY.

Uemura, T., S. Shepherd, L. Ackerman, L.Y. Jan, and Y.N. Jan. 1989. numb, a gene required in determination of cell fate during sensory organ formation in Drosophila embryos. Cell 58: 349-360.

Vaessin, H., E. Grell, E. Wolff, E. Bier, L.Y. Jan, and Y.N. Jan. 1991. prospero is expressed in neuronal precursors and encodes a nuclear protein that is involved in the control of axonal outgrowth in Drosophila. Cell 67: 941-953.

Webster, P.J., L. Liang, C.A. Berg, P. Lasko, and P.M . M acdonald. 1997. Translational repressor bruno plays multiple roles in development and is widely conserved. Genes \& Dev. 11: $2510-2521$.

Zhong, W., J.N . Feder, M .-M . Jiang, L.Y. Jan, and Y.N . Jan. 1996. Asymmetric localization of a mammalian numb homolog during mouse cortical neurogenesis. Neuron 17: 43-53. 


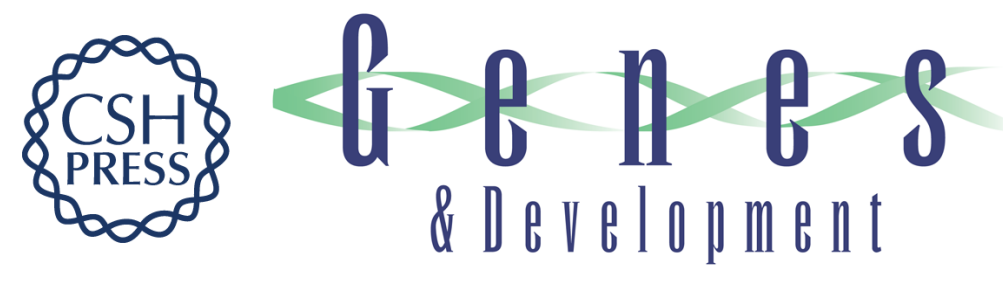

\section{Miranda mediates asymmetric protein and RNA localization in the developing nervous system}

Alison J. Schuldt, Jan H.J. Adams, Catherine M. Davidson, et al.

Genes Dev. 1998, 12:

Access the most recent version at doi:10.1101/gad.12.12.1847

References

This article cites 54 articles, 12 of which can be accessed free at: http://genesdev.cshlp.org/content/12/12/1847.full.html\#ref-list-1

License

Email Alerting

Receive free email alerts when new articles cite this article - sign up in the box at the top Service right corner of the article or click here.

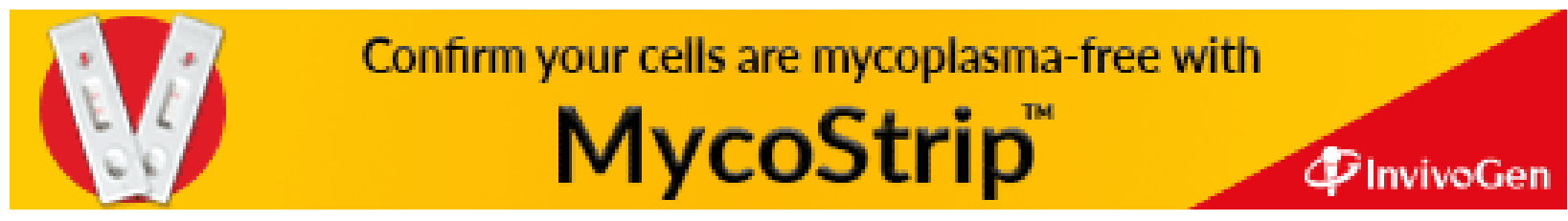

